\title{
Hallmarks of a secure psychiatric service for women
}

\author{
Charles Kaye
}

Dissatisfaction with current psychiatric services provided for women in secure heath environments is described. Mention is made of recent improvements in the spectal houplitals. A sot of key criteria is suggested to judge the effectiveness of services wherever provided.

\section{A mired inheritance}

The psychiatric service for women needing a secure environment has been the subject of considerable criticism. Dissatisfaction has been voiced by people on the outside looking in, by the recipients of the service (the women themselves) and by the staff who are involved in providing the service.

There have been three main components to that dissatisfaction and unease:

(a) anxiety about the standards of care which were inconsistent and not sufficiently well defined

(b) a strong feeling that the regimes of care failed to reflect the current values in society with regard to the women's changing roles, their needs and the realisation of their potential. The service seemed to be trapped within an outdated environment which was not sensitive to women's needs or the issues of dealing with seriously ill patients

(c) a concern, which although difficult to quantify, had both strength and validity, that the criminal justice system, when it was brought to bear on women who had offended or behaved in a disturbed fashion, was using different standards for assessing women from those applied to men. As a result women were often receiving harsher sentences for similar offences. Again this seemed to be based on a limiting concept of woman as conforming wife and mother; women who failed to conform to this 'ideal' were paying additional penalties.

Although each of these points can be challenged, there is strong evidence for the first two; circumstantial and anecdotal evidence for the third would bear more research.

\section{The challenge}

When the Special Hospitals Service Authority (SHSA) took over responsibility for the special hospitals in 1989, it needed to reshape its services for women to meet these criticisms. The immense currents around women and women's services were far from conducive to reasoned clinical care and a well balanced programme of treatment. Cross currents and tensions within the hospitals, the service and the staff, were getting in the way of decent care. Although much of this turbulence reflected wider issues in society, attention became especially focused on the special hospitals because of their particular role.

At the same time there were, and are, doubts being expressed about the appropriateness of medium secure units as a setting for treating disturbed women. These small, mainly male communities could be seen as antipathetic to the gender sensitive treatment and care required for women patients. The SHSA proposed, before its demise, an alternative national pattern of care for women.

Importantly, the cost of caring for women patients is high. In 1995-1996 they accounted for approximately $\$ 20$ million out of a total revenue budget for the SHSA of $£ 109$ million. In the same year the average annual cost for a woman patient in a special hospital was $£ 102000$ compared with $\$ 80000$ for a man.

\section{The response}

The first area to be tackled in the special hospitals was that of standards and attitudes. Standards were set in all areas, and once set they were used together with training, education and discussion, to help tackle attitudes of staff, individually and in groups.

The next thing to challenge was the physical environment. The SHSA spent \&15-20 million per annum over its six years of existence to provide a better environment for the patients. A 
significant proportion of this sum was spent specifically on facilities for women patients. Efforts were also made to enlarge the environment for women within each hospital, although there are still areas which are not as open to women as they are to men. Overall there has been considerable improvement with regard to the physical environment and access to facilities.

The third aspect to be tackled was the brief for the service itself. The authority launched a wide and open discussion, incorporating contributions from clinicians, staff and outside agencies. The strategy which has now been formulated represents the culmination of all that experience and expertise and lays down the fundamentals of care for women and suggests the most appropriate arrangements to provide that care and these standards. ('Service strategies for secure care' published by the SSHA. December 1995. Available from C. K. upon request.)

As a result of this work it has been possible to identify a number of key criteria or 'hallmarks' by which any service for women in a secure setting could be judged. The authority's experience suggests that any service, whether high or medium security, public or private sector, needs to perform well against each criterion to be successful.

\section{Service hallmarks}

Setting the scene

(a) An environment that provides a haven for women but is not isolated. This suggests units that are dedicated to women although they may be linked to similar men's units.

(b) A sympathetic approach in a gender sensitive atmosphere. Responding to each individual with the minimum of preconceptions.

(c) Demonstrable clinical philosophy of care. An approach to care and treatment which is consistent, agreed by all professions involved, and the essence of which is written down.

\section{Care and treatment}

(a) Active involvement of each woman in her care programme e.g. by sharing of the care plan and goals, attendance at case conferences.

(b) Explicit recognition of the significance of past traumatic experiences for the majority of the patients. This would form part of the philosophy of care and would describe techniques and responses.

(c) Clinical strategies that respond positively to, and speedily reduce, self-injurious behaviour. (See the interesting work in progress at Rampton and Ashworth Hospitals (Swinton \& Hopkins, 1996).)

(d) Specific treatment programmes for women with substance misuse problems and for those with eating disorders.

(e) Staffed by men and women who have all been trained in their roles in a service for women.

(f) Good and effective contacts with outstde networks.

\section{Demonstrating and validating}

(a) Well articulated criteria for clinical progress within the unit and afterwards measurement and recording of sucess, this is likely to be based on openly expressed goals within the care plans as well as longer-term follow up.

(b) A focus for targeted research which feeds back to modify and improve practice.

(c) The ability to cost and justify each treatment for each individual.

\section{Standards of care}

These interdependent standards could be summarised:

(a) providing an appropriate environment with the right atmosphere

(b) establishing a consistent philosophy and practice of care which could be demonstrated and which would be shared with and would focus on each individual patient. Philosophy and practice would meet identified needs and be responsive

(c) setting goals and measuring progress. Although this seems elementary, there are far too many instances where it is still not done. The different stages of clinical progress should be set and measured to demonstrate the progress. Progress which is claimed but which cannot be demonstrated is not convincing. A culture of research needs to permeate the service, particularly into areas which are clinically uncharted. As an integral part of contracting and purchasing, all the aspects of care also need to be linked to cost. It is important to analyse the allotment of the costs to ensure the best investment is made to produce results and achieve therapeutic goals.

\section{Conclusion}

This set of standards poses the key questions. This article does not seek to demonstrate how they can be answered. It is acknowledged that some of these requirements will make major demands on even the most dedicated clinical 
team, but this is not surprising when one considers the difficulty of providing effective treatment and support for these patients.

The set of criteria taken as an entity offer a practical template for assessing services by all concerned. They provide a well balanced basis for future discussion between all interested parties and might help to discard prejudice and preconceptions in favour of good demonstrable practice.

\section{Acknowledgements}

Many of these ideas were originally presented to a conference on 'Services for Women in Secure
Care' held in Telford in November 1995. My thanks to Dr Fiona Mason and Dr Dilys Jones for their helpful comments.

\section{Reference}

SWinton. M. \& Hopkins, R. (1996) Violence and self-injury. Journal of Forensic Psychiatry. 7, 563-569.

Charles Kaye (formerly Chief Executive of the Special Hospitals Service Authority) 18 Tanhouse Lane, Alton, Hampshire GU34 1 HR

\section{Prevention of Anxiety and Depression in Vulnerable Groups}

\section{Joanna Murray}

The scope of this review, commissioned by the Department of Health, is the common mental disorders of anxiety and depression occurring in adults in the community. It considers the possibilities for prevention in primary care. This combination of basic conceptual and research information provides a practical framework of preventive strategies for the primary care team. Social factors in aetiology are examined in detail, and epidemiological data is used to consider vulnerability factors and to identify high risk groups. There is also a thorough review of risk for common mental disorders. £7.50, 112pp., 1995, ISBN 0902241877

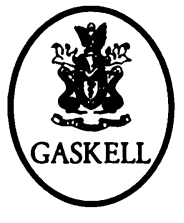

Gaskell books are available from good bookshops and from the Publications

Department, Royal College of Psychiatrists, 17 Belgrave Square, London SW1X 8PG (Tel. +44(0)171 2352351 , extension 146).

The latest information on College publications is available on the INTERNET at: http://www.demon.co.uk/rcpsych/ 